\title{
Chronic Helicobacter pylori Infection and DNA-Damaged Stem Cells: A Recipe for Disaster
}

\author{
Richard M. Peek Jr.
}

Published online: 31 October 2012

(c) Springer Science+Business Media New York 2012

Helicobacter pylori is the most common bacterial infection worldwide. Biological costs incurred by chronic colonization include increased risk for gastric adenocarcinoma [1]. Approximately 660,000 new cases of gastric cancer per year are attributable to $H$. pylori; eradication of this pathogen significantly decreases the risk of developing cancer in infected individuals. Nevertheless, only a small percentage of colonized persons develop neoplasia; enhanced risk is related to $H$. pylori strain differences, host responses governed by genetic diversity, and/or specific interactions between host and microbial determinants [2]. Universal test and treat strategies for $H$. pylori are not feasible due to the high prevalence of infection combined with the expense and side effects of antibiotic therapy [1]. These observations, in conjunction with evidence that carriage of certain strains is inversely related to esophageal adenocarcinoma and atopic diseases [1,3], underscore the importance of identifying mechanisms that regulate interactions of $H$. pylori with its human host which promote carcinogenesis.

One $H$. pylori strain-specific virulence locus that augments gastric cancer risk is the cag pathogenicity island, which encodes a type IV secretion system (TFSS) [4-6]. The product of the cagA gene ( $\mathrm{CagA})$ is translocated by the

R. M. Peek Jr.

Department of Medicine, Vanderbilt University, Nashville,

TN, USA

R. M. Peek Jr.

Department of Cancer Biology, Vanderbilt University,

Nashville, TN, USA

R. M. Peek Jr. (ه)

Division of Gastroenterology, Vanderbilt University,

1030C MRB IV, 2215B Garland Avenue, Nashville,

TN 37232-2279, USA

e-mail: richard.peek@vanderbilt.edu
TFSS into epithelial cells, undergoing targeted tyrosine phosphorylation by Src and Abl kinases at motifs (termed $\mathrm{A}, \mathrm{B}$, or $\mathrm{C} / \mathrm{D})$ containing the amino acid sequence EPIYA [7-10]. Phosphorylated CagA activates a cellular phosphatase (SHP-2) and ERK mitogen-activating protein kinases (MAPK) leading to morphological aberrations that mirror changes induced by growth factor stimulation [11, 12]. Moreover, non-phosphorylated intracellular CagA can exert effects with carcinogenic potential including activation of $\beta$-catenin [13, 14].

DNA damage resulting from inflammation-associated reactive oxygen and nitrogen species (RONS) also is contributory towards the development of pre-malignant lesions within $H$. pylori-infected gastric mucosa. Loss of a key enzyme, alkyladenine DNA glycosylase (AAG) that repairs DNA damage induced by RONS, augments the severity of atrophy and foveolar hyperplasia in the stomachs of mice challenged with $H$. pylori [15]. H. pylori can also directly induce DNA damage in gastric epithelial cells via activation of a pathway mediated by spermine oxidase (SMO). SMO catabolizes the formation of the polyamine spermine, which produces $\mathrm{H}_{2} \mathrm{O}_{2}$, leading to DNA damage [16]. Chaturvedi et al. [16] recently reported that $H$. pylori CagA can induce SMO-mediated DNA damage in vitro and in rodent models of infection, results that were mirrored by findings in human subjects colonized with $H$. pylori cag ${ }^{+}$ strains. Of great importance, $H$. pylori-infected rodents that developed dysplasia harbored a subset of gastric epithelial cells in which SMO production and DNA damage were high, but which were resistant to apoptosis, thereby representing a cellular population poised for neoplastic transformation (Fig. 1).

The gastric epithelium is organized into functionally monoclonal glandular units. Homeostasis is regulated via stem cells, which legislate glandular function via 
Fig. 1 Hypothetical model of DNA damage induced by intracellular delivery of $H$. pylori CagA into gastric stem cells

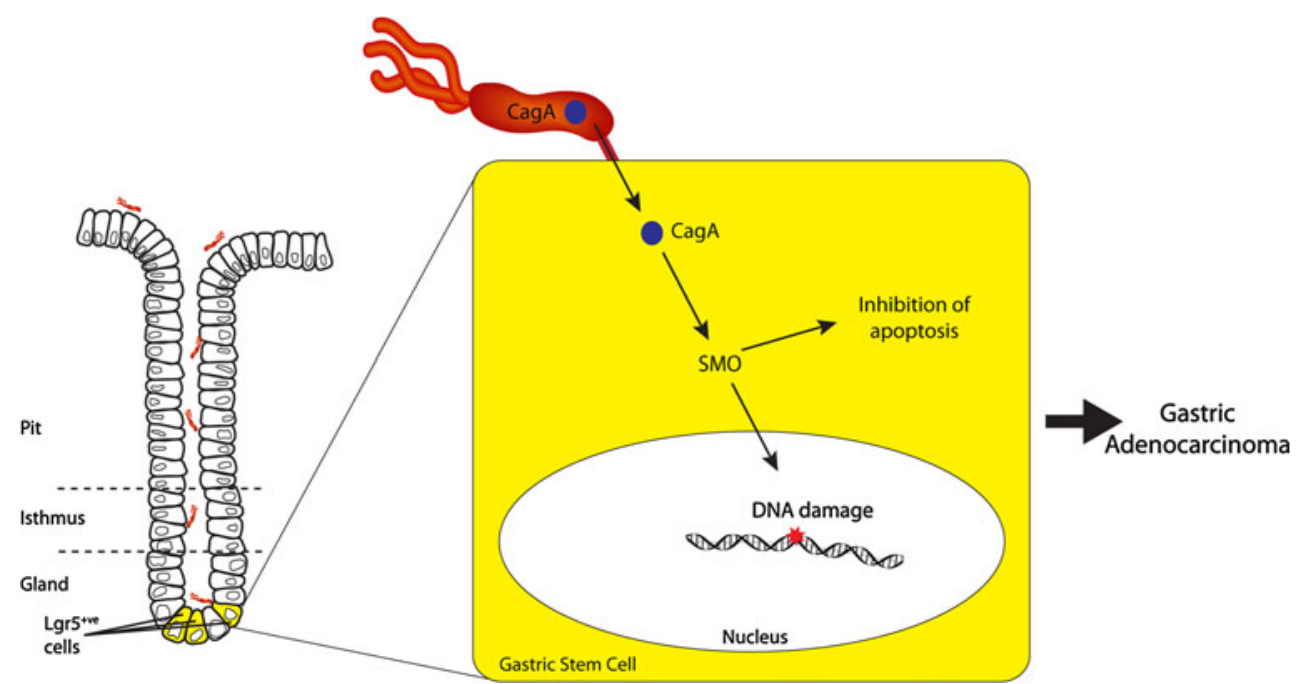

infrequent asymmetric divisions with subsequent differentiation of progeny into epithelial cell lineages. Stem cells are tightly regulated by Wnt proteins, which activate B-catenin. Barker et al. [17] previously demonstrated that the Wnt target gene Lgr5, encoding an orphan G proteincoupled receptor, marks a multipotent stem cell population responsible for long-term renewal of gastric epithelium. Further, a subpopulation of Lgr5-expressing cells possesses the ability to generate gastric organoid cultures in vitro, which could be maintained for up to 9 months [17].

Helicobacter pylori can interact intimately with stem cell populations. In transgenic mice that over-express $\mathrm{Le}^{\mathrm{b}}$, H. pylori adhere directly to gastric epithelial cells $[18,19]$. Genetic ablation of parietal cells in Le $\mathrm{Le}^{\mathrm{b}}$-expressing transgenic mice permits the gastric epithelial progenitor (GEP) stem cell population to expand, accompanied by an expansion of $H$. pylori colonization and inflammation within the glandular epithelium [20, 21]. Further, delineation of the GEP transcriptome has identified several pathways that are over-represented in this lineage which are of particular biological importance for carcinogenesis, including Wnt/B-catenin [22]. Epidemiologic data supporting the presence of aberrant $B$-catenin signaling in the majority of gastric malignancies, in conjunction with evidence that cancer-associated $H$. pylori $\mathrm{cagA}^{+}$strains activate this pathway, has fueled efforts to define how chronic inflammation mediates stem cell damage.

In this issue, Uehara et al. examined the relationship between $H$. pylori colonization, gastric cancer and DNA damage within $\mathrm{Lgr} 5^{+}$cells using specimens harvested from patients, an important approach since the human stomach represents the primary endogenous ecological niche for H. pylori. Lgr5-positive cells, as determined by immunohistochemistry and immunofluorescence, were primarily localized to the gastric antrum within the mucous neck region, without localization to surface or pit regions [23]. To bolster the specificity of Lgr5 as a stem cell marker, sections were also stained for another putative stem cell marker, CD44. Virtually all $\mathrm{Lgr} 5^{+}$cells also co-expressed CD44. To replicate these findings in an experimental in vivo system, Lgr5-positive cells were also identified within murine gastric mucosa where a similar topographical pattern of distribution was identified.

Gastric mucosa from colonized subjects with cancer contained significantly increased populations of $\operatorname{Lgr} 5^{+}$ cells, compared with uninfected patients, with cancer; however, Lgr5 ${ }^{+}$cell abundance was the same when all gastric cancer patients (infected and uninfected) were compared with all non-cancer patients. Thus, the presence of $H$. pylori influenced the expansion of stem cell populations in subjects who developed gastric malignancy.

Based upon these associations, the authors then delved into mechanisms that may underlie the development of cancer in these subjects. Buoyed by the finding of a strong relationship between the number of $\mathrm{Lgr}^{+}$cells and the severity of acute inflammation, Uehara et al. next quantified DNA damage using $8 \mathrm{OHdG}$ as a surrogate marker of oxidative DNA damage, stratifying their results based on Lgr5 cellular positivity. Similar to findings focused on Lgr5 per se, significantly higher levels of $8 \mathrm{OHdG}$ were detected in $\mathrm{Lgr}^{+}$epithelial cells compared with Lgr5negative cells among $H$. pylori-infected persons with gastric cancer. Of interest, within the lamina propria, levels of 8OHdG were higher in $\mathrm{Lgr}^{+}$cells in uninfected subjects who did not have gastric cancer. Further, in contrast to epithelial $\mathrm{Lgr}^{+}$cells, lamina propria $\mathrm{Lgr}^{+}$cells coexpressed CD45 and vimentin, suggesting a possible leukocyte lineage derivation. 
This study has offered important information regarding how a chronic bacterial infection may affect carcinogenesis within the context of inflammation, providing a framework for future studies focused on these relationships. As with all original research, new questions and directions for future experiments ensue from these results. Subsequent studies should focus on the microbial elements required for the induction of DNA damage in stem cells. As described previously, the translocated effector protein CagA can directly induce DNA damage in gastric epithelial cells (Fig. 1) [16]; thus a rational next step would be to stratify $H$. pylori-colonized persons into those that harbor $\mathrm{cagA}^{+}$versus $\mathrm{cagA} \mathrm{A}^{-}$ strains. In vitro studies can be performed to determine if the ability of CagA to induce DNA damage is dependent upon the phosphorylation status of translocated CagA. If so, CagA proteins from East Asian H. pylori strains, which are more potent in inducing cellular morphologic aberrations, could be compared to CagA proteins derived from Western H. pylori strains. A logical extension could incorporate a more detailed investigation of other stem cell populations that may be affected. In the intestine, Lgr5 marks a highly proliferative population of stem cells. In contrast, Powell et al. recently reported that Lrig1 (Leucine-rich repeats and immunoglobulin-like domains 1) is a transmembrane protein that marks a distinct population of quiescent stem cells which functions as a tumor suppressor [23]. Lrig1 is expressed in the stomach; therefore, it would be additionally important to define the effects of $H$. pylori infection on this stem cell population. Finally, studies of mice infected with $H$. pylori or with the related mouse-adapted Helicobacter species, H. felis, have demonstrated that bone marrow derived cells (BMDCs) home to and engraft in sites of chronic gastric inflammation, particularly within foci where tissue injury induces excessive apoptosis and overwhelms the population of endogenous tissue stem cells [24,25]. Within the inflamed stomach, BMDCs degenerate into adenocarcinoma, suggesting that gastric epithelial carcinomas can originate from marrow-derived sources $[24,25]$. Are the stem cells identified in the current study endogenous to the stomach or are they derived from other sites?

Studies such as the one performed by Uehara et al. are important in that they have utilized the natural niche of H. pylori as a nidus to demonstrate biologically relevant relationships between elements of the microbiota, host responses, and disease. Investigations that focus on specific interactions between $H$. pylori and its host can provide models for general patterns that may be extended to other malignancies that arise from inflammatory foci and facilitate a deeper understanding of how chronic inflammation leads to malignant degeneration.

Acknowledgments Grant support: NIH CA-116087, DK-58404, DK-58587, and CA-77955.

\section{References}

1. Polk DB, Peek RM Jr. Helicobacter pylori: gastric cancer and beyond. Nat Rev Cancer. 2010;10:403-414.

2. Ernst PB, Peura DA, Crowe SE. The translation of Helicobacter pylori basic research to patient care. Gastroenterology. 2006;130: 188-206.

3. Chen Y, Blaser MJ. Inverse associations of Helicobacter pylori with asthma and allergy. Arch Intern Med. 2007;167:821-827.

4. Crabtree JE, Wyatt JI, Sobala GM, et al. Systemic and mucosal humoral responses to Helicobacter pylori in gastric cancer. Gut. 1993;34:1339-1343.

5. Blaser MJ, Perez-Perez GI, Kleanthous H, et al. Infection with Helicobacter pylori strains possessing cagA is associated with an increased risk of developing adenocarcinoma of the stomach. Cancer Res. 1995;55:2111-2115.

6. Parsonnet J, Friedman GD, Orentreich N, Vogelman H. Risk for gastric cancer in people with CagA positive or CagA negative Helicobacter pylori infection. Gut. 1997;40:297-301.

7. Selbach M, Moese S, Hauck CR, Meyer TF, Backert S. Src is the kinase of the Helicobacter pylori CagA protein in vitro and in vivo. J Biol Chem. 2002;277:6775-6778.

8. Backert S, Moese S, Selbach M, Brinkmann V, Meyer TF. Phosphorylation of tyrosine 972 of the Helicobacter pylori CagA protein is essential for induction of a scattering phenotype in gastric epithelial cells. Mol Microbiol. 2001;42:631-644.

9. Tammer I, Brandt S, Hartig R, Konig W, Backert S. Activation of Abl by Helicobacter pylori: a novel kinase for CagA and crucial mediator of host cell scattering. Gastroenterology. 2007;132:13091319.

10. Mueller D, Tegtmeyer N, Brandt S, et al. c-Src and c-Abl kinases control hierarchic phosphorylation and function of the CagA effector protein in Western and East Asian Helicobacter pylori strains. J Clin Invest. 2012;122:1553-1566.

11. Higashi H, Nakaya A, Tsutsumi R, et al. Helicobacter pylori CagA induces Ras-independent morphogenetic response through SHP-2 recruitment and activation. J Biol Chem. 2004;279:1720517216.

12. Higashi H, Tsutsumi R, Muto S, et al. SHP-2 tyrosine phosphatase as an intracellular target of Helicobacter pylori CagA protein. Science. 2002;295:683-686.

13. Franco AT, Israel DA, Washington MK, et al. Activation of Bcatenin by carcinogenic Helicobacter pylori. Proc Natl Acad Sci USA. 2005;102:10646-10651.

14. Nagy TA, Wroblewski LE, Wang D, et al. Beta-Catenin and p120 mediate PPARdelta-dependent proliferation induced by Helicobacter pylori in human and rodent epithelia. Gastroenterology. 2011;141:553-564.

15. Meira LB, Bugni JM, Green SL, et al. DNA damage induced by chronic inflammation contributes to colon carcinogenesis in mice. J Clin Invest. 2008;118:2516-2525.

16. Chaturvedi R, Asim M, Romero-Gallo J, et al. Spermine oxidase mediates the gastric cancer risk associated with Helicobacter pylori CagA. Gastroenterology. 2011;141:1696-1708.

17. Barker N, Huch M, Kujala P, et al. Lgr5(+ve) stem cells drive self-renewal in the stomach and build long-lived gastric units in vitro. Cell Stem Cell. 2010;6:25-36.

18. Falk PG, Bry L, Holgersson J, Gordon JI. Expression of a human alpha-1,3/4-fucosyltransferase in the pit cell lineage of FVB/N mouse stomach results in production of $\mathrm{Le}^{\mathrm{b}}$-containing glycoconjugates: a potential transgenic mouse model for studying Helicobacter pylori infection. Proc Natl Acad Sci USA. 1995;92:1515-1519.

19. Guruge JL, Falk PG, Lorenz RG, et al. Epithelial attachment alters the outcome of Helicobacter pylori infection. Proc Natl Acad Sci USA. 1998;95:3925-3930. 
20. Syder AJ, Guruge JL, Li Q, et al. Helicobacter pylori attaches to NeuAc alpha 2,3Gal beta 1,4 glycoconjugates produced in the stomach of transgenic mice lacking parietal cells. Mol Cell. 1999;3:263-274.

21. Syder AJ, Oh JD, Guruge JL, et al. The impact of parietal cells on Helicobacter pylori tropism and host pathology: an analysis using gnotobiotic normal and transgenic mice. Proc Natl Acad Sci USA. 2003;100:3467-3472.

22. Oh JD, Kling-Backhed H, Giannakis M, et al. The complete genome sequence of a chronic atrophic gastritis Helicobacter pylori strain: evolution during disease progression. Proc Natl Acad Sci USA. 2006;103:9999-10004.
23. Uehara T, Ma D, Yao Y, et al. H. pylori infection is associated with DNA damage of Lgr5-positive epithelial stem cells in the stomach of patients with gastric cancer. Dig Dis Sci. 2012. (Epub ahead of print). doi:10.1007/s10620-012-2360-8.

24. Powell AE, Wang Y, Li Y, et al. The pan-ErbB negative regulator Lrig1 is an intestinal stem cell marker that functions as a tumor suppressor. Cell. 2012;149:146-158.

25. Houghton J, Stoicov C, Nomura S, et al. Gastric cancer originating from bone marrow-derived cells. Science. 2004;306:1568-1571.

26. Varon C, Dubus P, Mazurier F, et al. Helicobacter pylori infection recruits bone marrow-derived cells that participate in gastric preneoplasia in mice. Gastroenterology. 2012;142:281-291. 